\title{
Critical analysis of the potential for targeting STAT3 in human malignancy
}

This article was published in the following Dove Press journal:

OncoTargets and Therapy

29 July 2013

Number of times this article has been viewed

\section{Noah D Peyser \\ Jennifer R Grandis}

Departments of Otolaryngology and Pharmacology and Chemical Biology, University of Pittsburgh School of Medicine, Pittsburgh, PA, USA
Correspondence: Jennifer R Grandis Departments of Otolaryngology and Pharmacology and Chemical Biology, University of Pittsburgh School of Medicine, 203 Lothrop Street, Eye and Ear Institute Building, Suite 500, Pittsburgh, PA 15213, USA

$\mathrm{Tel}+\mathrm{I} 4 \mid 26475280$

Fax + I 4126472080

Email jgrandis@pitt.edu
Abstract: The signal transducer and activator of transcription (STAT) family of proteins was originally discovered in the context of normal cell biology where they function to transduce intracellular and extracellular signals to the nucleus, ultimately leading to transcription of specific target genes and downstream phenotypic effects. It was quickly appreciated that the STATs, especially STAT3, play a fundamental role in human malignancy. In contrast to normal biology in which transient STAT3 signaling is strictly regulated by a tightly coordinated network of activators and deactivators, STAT3 is constitutively activated in human malignancies. Constitutive STAT3 signaling has been associated with many cancerous phenotypes across nearly all human cancers, including the upregulation of cell growth, proliferation, survival, and motility, among others. Studies involving candidate preclinical STAT3 inhibitors have further demonstrated that the reversal of these phenotypes results from pharmacologic or genetic inhibition of STAT3, suggesting that STAT3 may be a promising target for clinical interventions. Indeed, a Phase 0 clinical trial involving a STAT3 decoy oligonucleotide demonstrated that STAT3 is a druggable target in human tumors. Because of the ubiquity of overactive STAT3 in cancer, its role in promoting a wide variety of cancerous phenotypes, and the strong clinical and preclinical studies performed to date, STAT3 represents a promising target for the development of inhibitors for the treatment of human cancers.

Keywords: cancer, target, STAT3, transcription factor

\section{Introduction}

The signal transducer and activator of transcription (STAT) family is a group of ubiquitously expressed proteins involved in a wide variety of cellular processes. Canonical STAT signaling involves STAT monomers localized in the cytoplasm where they receive a wide variety of specific upstream signals. Upon activation, STATs dimerize and translocate to the nucleus where they activate transcription of specific target genes, ultimately leading to altered protein expression and cellular phenotype. To date, seven STAT family members have been identified - STAT1, STAT2, STAT3, STAT4, STAT5A, STAT5B, and STAT6 - and multiple isoforms of each have also been found. Each STAT protein includes several conserved domains that contribute to protein function (Figure 1). The N-terminal protein-protein interaction domain (PPID) mediates interaction between neighboring STAT proteins (or other coregulatory proteins) and contributes to cooperative binding of STAT dimers on deoxyribonucleic acid (DNA), leading to the formation of stabilized tetramers. ${ }^{1}$ This function, while nonessential for transcriptional activation, may contribute to enhanced STAT3 signaling by prolonging DNA binding. The DNA-binding domain is involved in sequence-specific DNA 


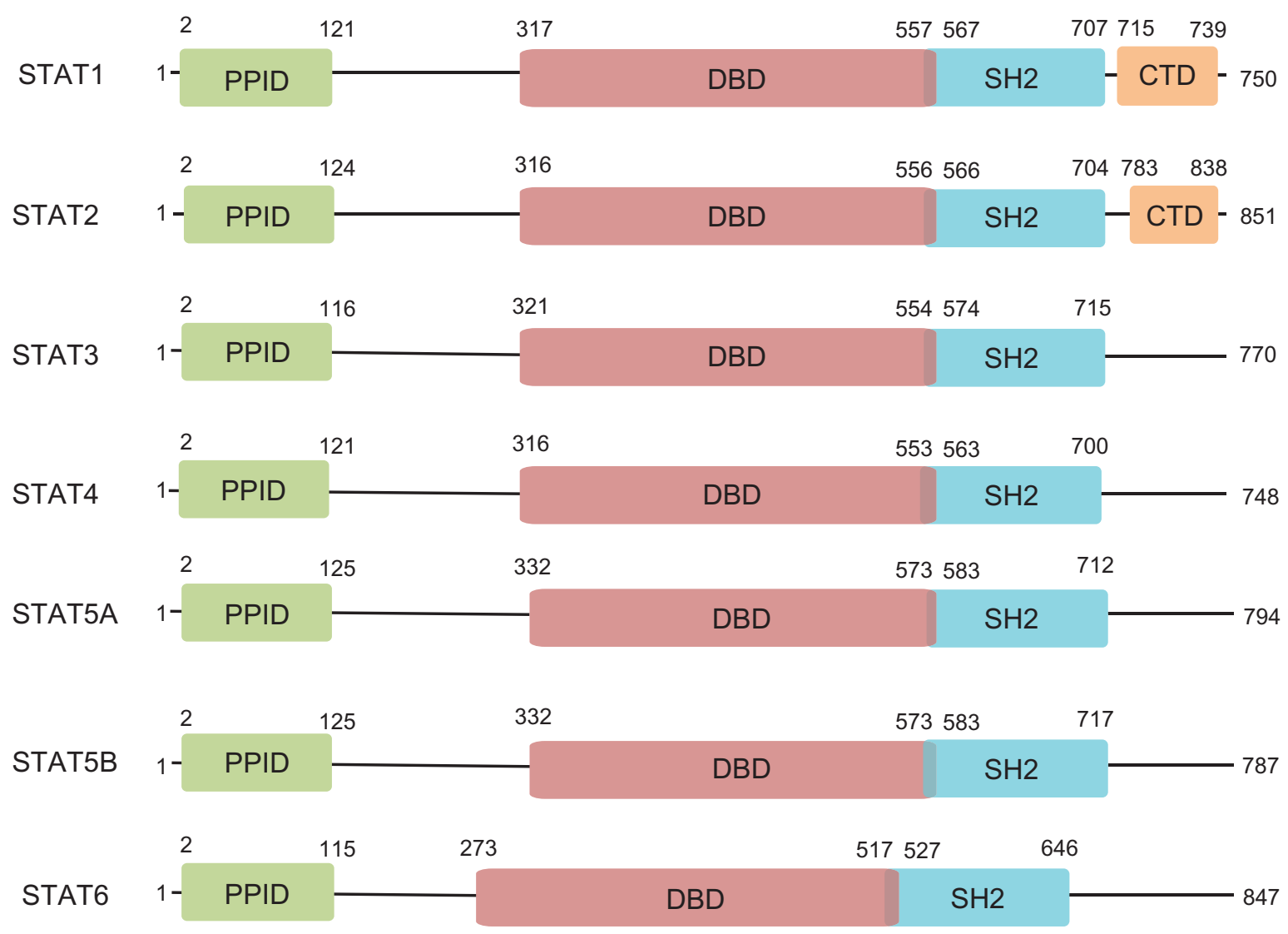

Figure I Domain architecture of STATs.

Notes: Members of the STAT protein family share similar domain structure, with each having an N-terminal PPID, a DBD, and an SH2. STATI and STAT2 also have a CTD that is involved in further protein-protein interactions. Numbers indicate amino acid positions as determined by the National Center for Biotechnology Information. "II

Abbreviations: STAT, signal transducer and activator of transcription; PPID, protein-protein interaction domain; DBD, DNA-binding domain; SH2, Src-homology 2 domain; CTD, C-terminal domain; DNA, deoxyribonucleic acid.

binding, recruitment of coactivators, and the activation of transcription of STAT3 target genes. The Src-homology 2 (SH2) domain is the mediator of STAT dimerization via reciprocal phosphotyrosine binding, a critical step for STAT activation. It is also involved in the recruitment of STAT to phosphotyrosine residues on other proteins, including tyrosine kinases, which then phosphorylate and activate STAT. The carboxy-terminal domains present in STAT1 and STAT2 are involved in further protein-protein interactions that impact STAT function, including those with comodulators of transcription such as the CREB binding protein. ${ }^{2}$ These domains coordinately determine the varied functions of each STAT protein.

STAT proteins were originally identified in the context of their physiological roles as major effectors of cytokine and chemokine receptor signaling. In recent years, the discovery that dysregulated STAT proteins are key modulators of human malignancy has driven research into the functions of these proteins. It now appears that the contribution of STAT proteins to cancer, especially overexpression and overactivation of STAT3, is crucial for the development and progression of many cancers. As such, STAT3 is a promising target for the development of inhibitors and is the focus of much ongoing research and drug development.

\section{Physiological roles of STAT3}

Phosphorylation of STAT3 on tyrosine 705 (Y705) by various upstream kinases is critical for STAT3 activation. ${ }^{3}$ A large number of protein tyrosine kinases directly phosphorylate STAT3 on Y705, including cytokine and chemokine receptors and their coactivators. Well-studied examples of such kinases include membrane integral receptor tyrosine kinases (RTKs) such as glycoprotein 130, the signaling subunit of the interleukin (IL)-6 receptor and other receptors, the epidermal growth factor receptor (EGFR), the fibroblast growth factor receptor, and non-RTKs that may or may not be associated with receptors such as Janus kinase (JAK), Src kinase, and Abl kinase. ${ }^{3-6}$ Upon STAT3 activation, dimers directly bind 
DNA at TT $(\mathrm{N})_{4-6} \mathrm{AA}$ consensus sites and regulate transcription of specific target genes. ${ }^{7}$ The binding affinity of STAT3 for this region is determined by both the nucleotide sequence and cooperative dimer-dimer interactions mediated by the amino-terminal PPID of STAT3., ${ }^{7.8}$ STAT3 activity can be further modulated by the phosphorylation of serine 727 , though the context-specific consequences of serine 727 phosphorylation remain incompletely understood. ${ }^{9,10}$ In addition, STAT3 activity can be modified by reversible acetylation of lysine 685 (K685), an event that is critical for the formation of stable STAT3 dimers and is required for cytokine-induced STAT3-mediated transcription. ${ }^{11,12}$

Downregulation of STAT3 occurs by several mechanisms in normal biology. The suppressor of cytokine signaling (SOCS) family of proteins, especially SOCS1 and SOCS3, inhibit STAT3 in a cytokine-inducible manner by binding to and inhibiting upstream JAKs. ${ }^{13,14}$ Members of the protein inhibitor of activated STAT (PIAS) family, especially PIAS3, are small ubiquitin-like modifier-E3 ligases that bind specifically to STAT3 and abrogate its activity. ${ }^{15}$ The SOCS and PIAS families together constitute major mechanisms by which STAT3 activity is downregulated quickly following stimulation with specific cytokines under normal conditions. Other proteins, including GRIM-19, can also abrogate STAT3 activity via direct interactions. ${ }^{16}$ Importantly, enzymatic removal of the phosphate group from Y705 of STAT3 by protein tyrosine phosphatases (PTPs) can also occur. Like the upstream kinases, PTPs that inactivate STAT3 can be membrane integral (PTPR family, including PTPRT and PTPRD) or cytosolic (PTPN family, including PTPN2 and PTPN11) ${ }^{17-20}$ Additionally, removal of the acetyl group from K685 of STAT3 by deacetylases, including SirT1, can lead to STAT3 downregulation. ${ }^{21,22}$ The intricacy and redundancy of the many mechanisms of STAT3 activation and deactivation illustrate the importance of maintaining tight control over the STAT3 pathway in normal biology.

Perhaps the field of normal biology in which STAT3 is most well studied is that of inflammation and the immune response. The actions of many cytokines and chemokines that led to the discovery of the STAT family, especially IL-6 and interferon (IFN), are mediated principally by STAT3 and are critical for proper immune function. STAT3 activation is triggered in epithelial tissue and associated macrophages in response to immunoglobulin $\mathrm{G}$ complex deposition or injury. ${ }^{23}$ In dendritic cells, the proinflammatory activity of IL-6 is mediated by transient activation of STAT3, whereas the antiinflammatory effects of IL-10 result from more sustained STAT3 activation. ${ }^{24}$ Interestingly, artificial early termination of IL-10 signaling leads to an IL-6-like cellular response. ${ }^{24}$ This rapid termination of IL-6 signaling appears to be mediated by SOCS3, which is encoded by a STAT3 target gene upregulated by both IL-6 and IL-10 that can inhibit signaling through the IL-6 receptor, but not the IL-10 receptor. ${ }^{24}$ These findings suggest that the divergent consequences of various signals upstream of STAT3 may in turn be determined by the contribution of STAT3 inactivators that ultimately determine the duration of STAT3 signaling.

Increased STAT3 activity is also associated with wound healing. As many of the genes involved in wound healing are also involved in oncogenesis, it is not surprising that STAT3 regulates many of the same genes in both of these processes. ${ }^{25}$ After cutaneous wounding in mice, IL-6 is upregulated in the epidermis primarily at the leading edge of the wound. ${ }^{26}$ Genetic knockout of $I L-6$ in mice leads to deficient cutaneous wound healing, with knockout mice requiring up to three-fold longer to heal than wild-type mice. ${ }^{26}$ Keratinocyte-specific STAT3 knockout in mice leads to impaired skin remodeling that results from impaired epidermal cell regeneration, confirming a central role for STAT3 in normal wound healing. ${ }^{27}$ In the gut, STAT3 activation in intestinal epithelial cells regulates immune homeostasis. ${ }^{28}$ Colonic $\mathrm{CD} 11 \mathrm{c}^{+}$cells secrete IL-22 in response to Toll-like receptor activation, leading to STAT3 activation in intestinal epithelial cells and promoting wound healing, demonstrating that STAT3 is essential for the wound healing process in a variety of tissues. ${ }^{28}$

STAT3 plays additional roles in several other normal cellular processes. For example, STAT3 functions as the downstream effector of important hormones such as insulin and leptin in both the brain and peripheral tissues, allowing for regulation of energy and metabolite homeostasis. ${ }^{29-32}$ STAT3 is also involved in autophagy, embryogenesis, proper thymic function, mammary development, and other processes. ${ }^{33-36}$ The importance of STAT3 activity in normal biology is demonstrated in part by the ubiquity of its tissue distribution. STAT3 activation across these tissues is a transient event, and STAT3 is quickly downregulated. When aberrations occur in the strict regulation of STAT3, malignancies can develop.

\section{Role of STAT3 in cancer Genomic and epigenomic deregulation of STAT3 in cancer}

The STAT3 protein is overexpressed and/or hyperactivated in the majority of human cancers. ${ }^{37}$ The prevalence of STAT3 overactivation in cancer cannot be explained by mutational activation of STAT3 because somatic mutation 
of the STAT3 gene in cancer is rare (1.08\%; 54/4980 tumors analyzed to date by whole exome sequencing by The Cancer Genome Atlas). ${ }^{38}$ Instead, STAT3 is the common effector of activating events affecting oncoproteins and deactivating events affecting tumor-suppressive proteins that ultimately lead to constitutive STAT3 activation. Dysregulation of diverse pathways that converge on STAT3 allows escape from the strict regulation that maintains transient STAT3 signaling in normal cell biology, leading to tumor-promoting cell proliferation, survival, motility, invasion, and angiogenesis. In addition, activation of STAT3 is associated with emergent resistance to targeted therapies and decreased patient survival. ${ }^{39,40}$

Among the first observations that indicated the importance of STAT3 in cancer was the phosphorylation of STAT3 by $\mathrm{V}$-Src - a known oncoprotein at that time - as well as constitutive STAT3 tyrosine phosphorylation and DNAbinding in several v-Src-transformed cell lines. ${ }^{41}$ Further study revealed that STAT3 activation and specific gene regulation is required for Src-mediated transformation of NIH-3T3 cells, leading to the conclusion that activation of STAT3 signaling is a critical component of malignant transformation. ${ }^{42,43}$ Additional studies generated similar findings in diverse systems, providing a strong case for the central role of STAT3 in a wide array of cancers. ${ }^{39,44-49}$ Years of continued research have convinced physicians and scientists of the significance of STAT3 in cancer and have elucidated many, though certainly not all, of the mechanisms by which aberrant STAT3 signaling contributes to malignancy.

In addition to Src kinase, many kinases upstream of STAT3 activation are frequently found to be altered in cancer cells, leading to constitutive kinase and STAT3 signaling. In neuroblastoma, frequent point mutation of the RTK anaplastic lymphoma kinase (ALK) in the kinase domain (F1174L) leads to constitutive activation of STAT3. ${ }^{50}$ Forced expression of this mutant, but not wild-type ALK, is sufficient to transform $\mathrm{Ba} / \mathrm{F} 3$ cells, enables cytokine-independent growth, and confers sensitivity to the small molecule ALK inhibitor TAE684 in neuroblastoma cell line models. ${ }^{50}$ Further, in ALK-positive anaplastic large-cell lymphoma cells that overexpress STAT3, inhibition of ALK leads to downregulation of total and active STAT3. ${ }^{51}$ Similar results have been found for other kinase domain mutations, including the wellstudied JAK2 mutation V617F, which is primarily found in myeloproliferative disorders. ${ }^{52,53}$ Activation of JAK2 caused by this mutation leads to constitutive activation of STAT3 and is associated with reduced survival in idiopathic myelofibrosis. ${ }^{54,55}$ Another mechanism of kinase-driven STAT3 activation in cancer is genomic amplification of kinase genes or RTK ligands with subsequent protein overexpression, leading to enhanced activation of wild-type kinases. For example, gene amplification of PDGFR $\alpha$ or EGFR in distinct subsets of glial tumors leads to enhanced expression of those proteins and downstream signaling events, including activation of STAT3 ${ }^{56}$ Overexpression of RTK ligands, such as IL-6 or transforming growth factor- $\alpha$, can also lead to persistent STAT3 activation via autocrine signaling through their receptors..$^{57,58}$ Other genomic events and rearrangements can also lead to kinase and STAT3 activation, such as that observed for the EGFRvIII protein, a constitutively active EGFR variant that is missing a large portion of the extracellular domain and exhibits impaired EGF binding. EGFRvIII expression is sufficient to transform NR6 cells (murine fibroblasts) and is associated with STAT3 activation and target gene expression. 59,60

Conversely, activation of STAT3 in human cancers can result from genomic or epigenomic inactivation of proteins that normally downregulate STAT3 activity. In contrast to the frequent activation of kinases by point mutation, deactivation of tumor-suppressive proteins by point mutation is relatively rare because of the necessity for such mutations to function in a dominant negative manner. These events do occur, however, as evinced by recently reported mutations in GRIM-19 that ablate its STAT3 inhibitory activity and promote tumor growth. ${ }^{16}$ Many investigators have recently begun to focus on epigenomic silencing of tumor-suppressive proteins that normally downregulate STAT3, especially by promoter hypermethylation. In lung cancer, for example, SOCS3 is frequently downregulated by promoter hypermethylation, and restoration of SOCS3 expression in cells where it was previously silenced leads to downregulation of active STAT3, induction of apoptosis, and suppression of cell growth. ${ }^{61}$ As SOCS proteins have not been demonstrated to inhibit kinases other than JAKs, inactivation of the SOCS family is unlikely to contribute substantially to aberrant STAT3 signaling across cancer types. Indeed, SOCS1 is unable to inhibit STAT3-mediated transformation of NIH-3T3 cells by v-Src and does not reduce STAT3 target gene expression in this system. ${ }^{62}$ Abnormal epigenomic alteration of other proteins that normally cause direct inactivation of STAT3, especially PTPs, remains incompletely understood and warrants further study. For instance, frequent methylation in the promoter region of PTPN6 is strongly correlated with decreased PTPN6 messenger ribonucleic acid (mRNA) expression and increased pSTAT3 expression in immunodeficiencyrelated non-Hodgkin lymphoma, demonstrating that 
epigenetic silencing of a phosphatase targeting pSTAT3 can lead to STAT3 activation. ${ }^{63}$ Other phosphatases that act upon pSTAT3, including PTPRD and PTPRT, have recently been reported to have tumor-suppressive functions and are frequently found to be altered in cancer cells, including, by promoter methylation. ${ }^{18,64}$ Additionally, the glutathione S-transferase family member GSTP1, which downregulates EGF-mediated STAT3 signaling and expression of STAT3 target genes via a direct interaction with STAT3, is promoterhypermethylated in hepatitis B virus-associated hepatocellular carcinoma and prostate cancer, and is subsequently downregulated. ${ }^{65-67}$

The diversity of genomic and epigenomic alterations in both activators and deactivators of STAT3 signaling is, in part, responsible for the high degree of difficulty in developing therapeutics that are applicable to a wide array of cancers; this suggests that targeting STAT3 directly may prove more efficacious. The further understanding of the many mechanisms contributing to aberrant STAT3 pathway activation may lead to the identification of biomarkers that can be used to establish subsets of patients who will most likely to respond to STAT3 inhibition.

\section{STAT3 in cell growth and proliferation}

STAT3 is a critical driver of cell growth in cancer, but not in normal cells. ${ }^{68}$ Constitutive STAT3 signaling has been implicated in aberrant cell growth and proliferation in many cancers, including head and neck squamous cell carcinoma, colorectal carcinoma, melanoma, glioblastoma multiforme, multiple myeloma, non-small cell lung cancer, and others. ${ }^{58,69-73}$ A critical mediator of cell growth downstream of STAT3 is its target CCND1, encoding gene cyclin D1, which is upregulated transcriptionally by active STAT3 and is required for STAT3-mediated transformation. ${ }^{74}$ Cyclin D1, in turn, acts through cyclin-dependent kinase (cdk)-dependent and cdk-independent mechanisms to allow passage through the G1 checkpoint of the cell cycle, ultimately leading to continuous and unregulated cell growth and proliferation. ${ }^{75} \mathrm{In}$ addition, the STAT3 target gene MYC, which itself encodes a transcription factor, is also a potent promoter of cell growth and is required for Src-mediated cellular transformation via STAT3. ${ }^{45}$

Other target genes of STAT3 that contribute to cell growth and proliferation include cytokines and growth factors that often act in an autocrine manner to further increase STAT3 signaling and/or other mitogenic pathways. It has recently come to be appreciated that nontraditional gene products, including micro-RNA molecules that downregulate specific genes by binding to specific mRNA transcripts, are also mediators of STAT3 mitogenic function. For example, STAT3 is persistently active in Wilms' tumor, a genetically heterogeneous childhood kidney cancer, where it transcriptionally upregulates the microRNA miR-370, which in turn regulates cell proliferation and tumorigenicity in mice. ${ }^{76}$ Cells transfected with miR-370 exhibit downregulation of the tumor suppressor WTX via direct binding to the 3 '-untranslated region of WTX mRNA, leading to its degradation. ${ }^{76}$ These cells also exhibit downregulation of the proteins $\mathrm{p} 21^{\mathrm{Cip} 1}$ and $\mathrm{p} 27^{\mathrm{Cip} 1}$ (which inhibit progress through the cell cycle) and upregulation of cyclin D1, illustrating novel mechanisms downstream of STAT3 that contribute to its proliferative capacity. ${ }^{76}$

\section{STAT3 in apoptosis and cell survival}

Constitutive STAT3 activation leads to evasion of apoptosis and a subsequent increase in cell survival. STAT3 transcriptionally regulates several Bcl-2 family members, including the antiapoptotic proteins $\mathrm{Bcl}-\mathrm{xL}, \mathrm{Bcl}-2$, and $\mathrm{Mcl}-1 .{ }^{77}$ The Bcl-2 family regulates apoptosis via homodimerization/ heterodimerization (the dynamics of which are determined stoichiometrically) and translocation to the mitochondrial membrane, where they ultimately regulate cytochrome c release and the initiation of apoptosis. STAT3-mediated upregulation of $\mathrm{Bcl}-\mathrm{xL}, \mathrm{Bcl}-2$, and $\mathrm{Mcl}-1$ contributes to apoptosis evasion in several cancers. ${ }^{77-79}$ STAT3-mediated Bcl-2 expression in metastatic subclones of the parental cell line MDA-MB435 (estrogen receptor-negative breast cancer) correlates with increased pSTAT3, but not with other transcription factors that regulate $\mathrm{Bcl}-2$, and contributes to chemoresistance in this cell line, suggesting that the antiapoptotic effects of STAT3 contribute to treatment sensitivity. ${ }^{80}$ Furthermore, a small peptide, ST3-H2A2, that inhibits the function of the N-terminal PPID of STAT3 induces the expression of multiple proapoptotic genes (and others) in prostate cancer cells, suggesting that STAT3 inhibition may restore normal apoptosis. ${ }^{81}$

STAT3 target genes that are not themselves in the Bcl-2 family can also contribute to evasion of apoptosis. Octamer transcription factor-1 (Oct-1) has been reported to be a target gene of STAT3 in esophageal squamous carcinoma cells (Eca-109), where STAT3 and Oct-1 coordinately regulate apoptosis. ${ }^{39}$ In these cells, activation of STAT3 by IL-6 treatment suppresses apoptosis as assessed by TUNEL staining, and knockdown of either STAT3 or Oct-1 by RNA interference enhances apoptosis. ${ }^{39}$ Conversely, forced overexpression of Oct-1 - even in the presence of STAT3 
knockdown - is sufficient to reduce apoptosis to similar levels as IL-6 treatment, suggesting that STAT3-driven Oct-1 expression may be sufficient to reduce apoptosis to minimal levels in these cells. ${ }^{39}$ STAT3 and Oct-1 knockdown leads to increased expression of proapoptotic Bax and Bad proteins, cytochrome c release from mitochondria, subsequent cleavage of caspases 3 and 9, and decreased expression of antiapoptotic Bcl-2 and Bcl-xL proteins. ${ }^{39}$ These findings provide a mechanism by which STAT3 overactivation leads to positive feedback in the suppression of apoptosis in conjunction with its target gene Oct-1.

\section{STAT3 in migration and invasion}

Constitutively active STAT3 further contributes to the cancer phenotype by promoting motility and invasion, including in human melanoma where increased activation of STAT3 promotes metastasis to the brain. ${ }^{82}$ The metastatic action of STAT3 is, in part, mediated by matrix metalloproteinases, a family of zinc-dependent endopeptidases that are secreted into the extracellular matrix. There the matrix metalloproteinases degrade extracellular matrix proteins, leading to facilitated cell migration, invasion through the basement membrane, and ultimately to the establishment of metastatic secondary tumors. The STAT3 target genes $M M P-2$ and $M M P-9$ are upregulated in esophageal squamous carcinoma cells (Eca-109) that express high pSTAT3, and STAT3 knockdown by RNA interference in these cells leads to downregulation of MMP-2 and MMP-9, dysregulation of cell migration directionality, decreased migration speed, and disorganization of F-actin formation; this demonstrates a central role for STAT3 in MMP-2/9-mediated cell motility. ${ }^{39}$ In addition, activation of STAT3 is required for maximal MMP-1 and MMP-10 induction in response to EGF in T24 bladder cancer cells, where STAT3 is a critical mediator of malignant characteristics. ${ }^{83}$

Other mechanisms that contribute to STAT3-mediated cell migration have been elucidated. For instance, EGFR activation via autocrine signaling in near-confluent, but not sparse, squamous cell carcinoma cells leads to activation of STAT3 and subsequent overexpression of the transmembrane glycoprotein podoplanin (PDPN). ${ }^{84}$ This cell density-regulated PDPN expression leads to increased cell migration and invasion, and these effects are reversed by shRNA knockdown of PDPN. ${ }^{84}$ Importantly, the observation of increased PDPN extends to clinical samples, in which PDPN is overexpressed in basal cell layers at the invading front of in situ SCC lesions, providing an additional clinically relevant mechanism by which STAT3 contributes to motility and invasion. ${ }^{84}$ Similarly, STAT3 is necessary for EGFR-mediated migration and invasion in prostate carcinoma cells. ${ }^{85}$ In addition, shRNA knockdown of STAT3 in Tu-2449 glioma cells leads to decreased PDPN expression and microvilli formation relative to vector-infected cells. ${ }^{86}$ Thus, inhibition of STAT3 may be an effective strategy for preventing malignant transformation and metastasis in several human cancers.

\section{STAT3 in the tumor microenvironment}

STAT3 is a critical regulator of the tumor microenvironment. For example, STAT3 is the downstream effector of several cytokine receptors that are involved in promoting angiogenesis, including those for vascular endothelial growth factor (VEGF), basic fibroblast growth factor, leptin, IL-6, and granulocyte-macrophage colony-stimulating factor. ${ }^{87-90}$ In addition, STAT3 can promote transcription of proangiogenic factors, including vascular endothelial growth factor and IL-6, leading to paracrine and/or autocrine feedback. ${ }^{91-93}$ Cytokine excretion from tumor cells also acts upon neighboring endothelial cells to promote proliferation, migration, and microvascular tube formation, leading to the development of mature blood vessels. The contribution of STAT3 activation to tumor angiogenesis, both in tumor cells and in endothelial cells, suggests that the inhibition of STAT3 may be an efficient method to block angiogenesis and tumor progression.

STAT3 is also involved in inflammation-associated carcinogenesis, suppression of the antitumor immune response, and maintenance of cancer stem cells. For example, nuclear factor-kappa B-mediated expression of IL-6 and subsequent activation of STAT3 is required for survival and evasion of apoptosis in intestinal epithelial cells during the development of colitis-associated cancer, a serious morbidity of irritable bowel disease. ${ }^{94,95}$ Furthermore, STAT3 activity is associated with reduced T-cell infiltration in isogenic murine melanomas, suggesting a role for STAT3 in suppressing tumor immunity. ${ }^{96}$ Inhibition of STAT3 in these tumor cells and also in glioblastoma cell models stimulates secretion of soluble factors, including TNF- $\alpha$ and IFN- $\beta$, that ultimately lead to increased infiltration of lymphocytes, natural killer cells, neutrophils, and macrophages, and also activates nitric oxide production from macrophages in vivo and in vitro. ${ }^{96,97}$ In addition, genetic or pharmacologic inhibition of STAT3 in glioblastoma stem cells, even transiently, leads to a loss of multipotency and an irreversible growth arrest, suggesting that STAT3 is required for maintenance and 
proliferation of cancer stem cells in this system. ${ }^{98}$ Thus, several mechanisms exist by which STAT3 inhibition may lead to tumor microenvironment disruption and subsequent regression.

\section{Overview of the current STAT3 inhibitors in clinical development}

Table 1 summarizes the STAT3 inhibitors that are currently in clinical development according to www.clinicaltrials.gov. Of the four trials listed, one is complete and three are recruiting or ongoing. Both the Isis (Isis Pharmaceuticals, Inc, Carlsbad, CA, USA) and AstraZeneca (London, UK) compounds are antisense oligonucleotide inhibitors of STAT3, whereas the Otsuka (Otsuka Pharmaceutical Development and Commercialization, Inc., Rockville, MD, USA) compound is a small molecule that downregulates STAT3 through an unknown mechanism of action. No results have yet been published from any of these three studies. Preclinical data recently reported in a poster abstract at the 2013 Annual Meeting of the American Association for Cancer Research demonstrate potent and selective downregulation of STAT3 mRNA and protein following AZD9150 treatment in several murine models, including human tumor xenografts. Downregulation resulted in strong antitumor activity and the study suggests that AZD9150 may be effective clinically. ${ }^{99}$

A full report of the completed STAT3 decoy Phase 0 trial and further development has been published. ${ }^{100}$ The decoy, which was designed to bind to the STAT3 DNA-binding domain and prevent STAT3 binding to chromatin, consists of a 15-mer duplex oligonucleotide with phosphorothioate caps at the $5^{\prime}$ and $3^{\prime}$ ends to enhance stability in vivo. Intratumoral injection of this molecule prior to surgical resection in head and neck squamous cell carcinoma (HNSCC) patients led to decreased expression of STAT3 target genes relative to saline-injected tumors in a Phase 0 trial, confirming the ability of the decoy to downregulate STAT3 signaling in human tumors. Systemic administration of the decoy in a murine xenograft model failed to demonstrate any effect on tumor growth or STAT3 signaling as a result of low stability of the decoy in serum. To overcome this difficulty, modified decoys were designed and tested. A circularized decoy consisting of the original decoy with two hexaethylene glycol linkages demonstrated enhanced stability in serum, with detectable levels observed for up to 12 hours. Importantly, systemic administration by intravenous injection of the cyclic decoy in murine xenograft models inhibited tumor growth and expression of STAT3 target genes, demonstrating a successful strategy to inhibit intratumoral STAT3 signaling via systemic, rather than intratumoral, administration. The cyclic decoy has not yet been tested in humans; efforts are underway to further improve its preclinical pharmacodynamic and pharmacokinetic parameters.

In addition to targeting STAT3 via its DNA-binding domain with an oligonucleotide decoy, STAT3 may be targeted via its $\mathrm{SH} 2$ domain by small molecules, peptides, or peptidomimetic compounds. ${ }^{101-104}$ Such molecules are designed to disrupt STAT3 dimerization, thus preventing its translocation to the nucleus and transcription. Other inhibition strategies include the introduction of antisense oligonucleotides, as in the case of the Isis Pharmaceuticals, Inc/AstraZeneca drug in clinical development, designed to cause degradation of STAT3 mRNA or prevent its translation via complementary base pairing, thereby reducing total STAT3 protein levels. ${ }^{105}$ Antisense strategies in particular will require exquisite tissue specificity, as they may lead to underexpression of STAT3 in normal tissues where its function is required. Recent high throughput and in silico

Table I STAT3 inhibitors currently in clinical development according to www.clinicaltrials.gov

\begin{tabular}{|c|c|c|c|c|c|c|}
\hline Drug name & Phase & Condition & STAT3 targeting strategy & Sponsor & Status & Reference \\
\hline STAT3 decoy & 0 & HNSCC & DBD oligonucleotide decoy & University of Pittsburgh & Complete & 100 \\
\hline ISIS-STAT3 $3_{R x}$ & $\mathrm{I} / 2$ & $\begin{array}{l}\text { Advanced cancers, } \\
\text { DLBCL, lymphoma }\end{array}$ & Antisense oligonucleotide & $\begin{array}{l}\text { Isis Pharmaceuticals, Inc } \\
\text { (Carlsbad, CA, USA) }\end{array}$ & Recruiting & \\
\hline AZD9I50 & 1 & $\begin{array}{l}\text { Advanced adult hepatocellular } \\
\text { carcinoma, hepatocellular } \\
\text { carcinoma metastatic }\end{array}$ & Antisense oligonucleotide & $\begin{array}{l}\text { AstraZeneca } \\
\text { (London, UK) }\end{array}$ & $\begin{array}{l}\text { Not yet } \\
\text { recruiting }\end{array}$ & \\
\hline OPB-51602 & 1 & Advanced cancer & $\begin{array}{l}\text { Small molecule, } \\
\text { target unknown }\end{array}$ & $\begin{array}{l}\text { Otsuka Pharmaceutical } \\
\text { Development and } \\
\text { Commercialization, Inc } \\
\text { (Rockville, MD, USA) }\end{array}$ & $\begin{array}{l}\text { Ongoing, } \\
\text { not recruiting }\end{array}$ & \\
\hline
\end{tabular}

Abbreviations: STAT3, signal transducer and activator of transcription 3; HNSCC, head and neck squamous cell carcinoma; DBD, deoxyribonucleic-binding domain; DLBCL, diffuse large B-cell lymphoma. 
screens also have the potential to identify novel strategies for targeting STAT3. ${ }^{101,106}$

\section{Critical analysis of the potential for the use of STAT3 inhibitors in the management of human malignancy}

For any protein to be the optimal target of inhibition for cancer treatment, it must exhibit several characteristics. Inhibition of the target protein must lead to downregulation of cell growth/proliferation, motility/invasion, and angiogenesis, as well as upregulation of apoptosis, cell death, and the antitumor immune response. The ideal target would also be applicable across a wide variety of cancer types. The inhibition of STAT3 in preclinical models has demonstrated all these characteristics across a wide variety of cancers, most likely via reversal of the many mechanisms discussed previously in this review. This suggests that STAT3 may be the ultimate target for inhibition in human malignancy. Importantly, a Phase 0 trial has demonstrated that STAT3 can be effectively targeted in human tumors, and further preclinical studies have suggested that systemic delivery of STAT3 inhibitors is likely to be effective. ${ }^{100}$ In addition, because STAT3 signaling is transient in normal tissues and cells, the potential for adverse events following systemic administration of a STAT3 inhibitor is minimal. Indeed, toxicology studies in nonhuman primates demonstrate a lack of toxicity of a STAT3 decoy oligonucleotide. ${ }^{107}$

Many of the recently approved cancer therapies target tyrosine kinases that are upstream of STAT3 activation, among other pathways. One hypothesis is that mutations in these kinases would signify constitutive activation and serve as a biomarker for patients who will most likely respond to these therapies. Unfortunately, clinical success with these agents has been limited, although in some cases they do prove extremely effective. Studies in preclinical models demonstrate that non-small cell cancer cell lines with mutations in select tyrosine kinases do not exhibit decreased STAT3 activation upon treatment with the respective targeted small molecules erlotinib (EGFR), U0126 (MEK1/2), sunitinib (PDGFRA), or crizotinib (MET), though other downstream effects of these inhibitors, including downregulation of phosphatidylinositol 3-kinase signaling, do occur. ${ }^{108}$ These findings support the notion that directly targeting STAT3, rather than any large number of its upstream activators, may be more efficacious in reversing the effects of constitutive STAT3 signaling.
Though some patients initially respond to targeted therapies, many develop chemoresistance and secondary cancers that are associated with increased STAT3 signaling. For example, overactivation of STAT3 is associated with resistance to EGFR-targeted therapies in several cancers, including HNSCC, bladder cancer, and others. ${ }^{40,80}$ Biopsies of recurrent HNSCC following treatment with cetuximab, a Food and Drug Administration-approved monoclonal antibody targeting EGFR, exhibit elevated pSTAT3 relative to pretreatment samples. These results suggest that STAT3 inhibition may be effective at overcoming acquired resistance or as adjuvant therapy to prevent recurrence. ${ }^{40}$ Treatment of bladder cancer cell lines that are resistant to cetuximab and exhibit elevated levels of activated STAT3 relative to cetuximab-sensitive cell lines leads to reduced cell viability and downregulation of STAT3 target genes. ${ }^{40}$ Importantly, a combination of STAT3 inhibition with EGFR blockade significantly enhances antitumor effects in vivo relative to EGFR blockade alone, suggesting that the efficacy of already existing (and approved) drugs may be significantly increased by concomitant treatment with STAT3 inhibitors. ${ }^{40}$ An additional mechanism of acquired resistance to targeted therapy is the activation of IL-6 following treatment. For example, acquired resistance to trastuzumab (a monoclonal antibody targeting the HER2/neu receptor) in HER2-positive breast cancer is associated with the activation of an IL-6 inflammatory feedback loop in which downstream STAT3 signaling contributes to cancer stem cell proliferation, providing additional rationale for cotargeting with a STAT3 inhibitor. ${ }^{109}$ Likewise, resistance to the tyrosine kinase inhibitor imatinib mesylate, which targets the BCR-ABL oncoprotein, in chronic myeloid leukemia cell models is also associated with increased STAT3 activation and target gene expression. ${ }^{110}$ Knockdown of STAT3 by small interfering RNA in this context resensitizes the cells to imatinib mesylate-induced cell death, suggesting that STAT3 inhibition may also be effective at overcoming targeted therapy resistance in hematological malignancies. ${ }^{110}$

Challenges in the development of STAT3 inhibitors to date have largely been overcome. Firstly, STAT3 and other transcription factors were widely regarded as untreatable with drugs mainly because they are not exposed to the extracellular surface and do not have clear ligand-binding domains that can be targeted for competitive inhibition. These perceived difficulties have proven surmountable in the case of STAT3 in both preclinical and clinical models, with STAT3-targeting agents effectively downregulating the pathway and reversing its oncogenic effects. A second 
obstacle in targeting STAT3 was its structural homology with STAT1, a family member with tumor-suppressive properties in many systems. This challenge has been overcome both with oligonucleotide inhibitors, which exploit the exquisite specificity of the DNA-binding domain, and with recent high-throughput screens of compound libraries to identify candidates that specifically inhibit STAT3 and not STAT1. Both of these strategies may lead in the near future to novel clinical therapeutics targeting STAT3.

\section{Conclusion}

Recent advances in the understanding of STAT3 signaling and its role in cancer have led to the establishment of STAT3 as a potential target for a wide variety of human malignancies. Whereas some clinical success has been found in the treatment of cancer with nonspecific chemotherapeutics and some targeted agents, there remains an urgent need for new classes of inhibitors of novel targets that will be widely applicable, well tolerated, and highly effective. The sum of preclinical and clinical data to date supports a unique role for STAT3 as one such target. Indeed, many therapeutic clinical successes to date have been associated with decreased STAT3 signaling, but because of the diversity of signaling components upstream of STAT3, the high cost of developing inhibitors for each one, the potential for emergent compensatory mechanisms, and the development of resistance to a given therapy, directly targeting STAT3 will likely be a preferred strategy.

\section{Disclosure}

The authors report no conflicts of interest in this work.

\section{References}

1. Vinkemeier U, Cohen SL, Moarefi I, Chait BT, Kuriyan J, Darnell JE Jr. DNA binding of in vitro activated Stat 1 alpha, Stat 1 beta and truncated Stat1: interaction between NH2-terminal domains stabilizes binding of two dimers to tandem DNA sites. EMBO J. 1996;15(20):5616-5626.

2. Wojciak JM, Martinez-Yamout MA, Dyson HJ, Wright PE. Structural basis for recruitment of $\mathrm{CBP} / \mathrm{p} 300$ coactivators by STAT1 and STAT2 transactivation domains. EMBO J. 2009;28(7):948-958.

3. Zhong Z, Wen Z, Darnell JE Jr. Stat3: a STAT family member activated by tyrosine phosphorylation in response to epidermal growth factor and interleukin-6. Science. 1994;264(5155):95-98.

4. Akira S, Nishio Y, Inoue M, et al. Molecular cloning of APRF, a novel IFN-stimulated gene factor 3 p91-related transcription factor involved in the gp130-mediated signaling pathway. Cell. 1994;77(1): 63-71.

5. Hart KC, Robertson SC, Kanemitsu MY, Meyer AN, Tynan JA, Donoghue DJ. Transformation and Stat activation by derivatives of FGFR1, FGFR3, and FGFR4. Oncogene. 2000;19(29):3309-3320.

6. Cirri P, Chiarugi P, Marra F, et al. c-Src activates both STAT1 and STAT3 in PDGF-stimulated NIH3T3 cells. Biochem Biophys Res Commun. 1997;239(2):493-497.

7. Ehret GB, Reichenbach P, Schindler U, et al. DNA binding specificity of different STAT proteins. Comparison of in vitro specificity with natural target sites. J Biol Chem. 2001;276(9):6675-6688.
8. Xu X, Sun YL, Hoey T. Cooperative DNA binding and sequenceselective recognition conferred by the STAT amino-terminal domain. Science. 1996;273(5276):794-797.

9. Andrés RM, Hald A, Johansen C, Kragballe K, Iversen L. Studies of Jak/STAT3 expression and signalling in psoriasis identifies STAT3Ser727 phosphorylation as a modulator of transcriptional activity. Exp Dermatol. 2013;22(5):323-328.

10. Wakahara R, Kunimoto H, Tanino K, et al. Phospho-Ser727 of STAT3 regulates STAT3 activity by enhancing dephosphorylation of phosphoTyr705 largely through TC45. Genes Cells. 2012;17(2):132-145.

11. Wang R, Cherukuri P, Luo J. Activation of Stat 3 sequence-specific DNA binding and transcription by $\mathrm{p} 300 / \mathrm{CREB}-$ binding protein-mediated acetylation. J Biol Chem. 2005;280(12):11528-11534.

12. Yuan ZL, Guan YJ, Chatterjee D, Chin YE. Stat3 dimerization regulated by reversible acetylation of a single lysine residue. Science. 2005;307(5707):269-273.

13. Sasaki A, Yasukawa H, Suzuki A, et al. Cytokine-inducible SH2 protein-3 (CIS3/SOCS3) inhibits Janus tyrosine kinase by binding through the N-terminal kinase inhibitory region as well as $\mathrm{SH} 2$ domain. Genes Cells. 1999;4(6):339-351.

14. Yasukawa H, Misawa H, Sakamoto H, et al. The JAK-binding protein $\mathrm{JAB}$ inhibits Janus tyrosine kinase activity through binding in the activation loop. EMBO J. 1999;18(5):1309-1320.

15. Chung CD, Liao J, Liu B, et al. Specific inhibition of Stat3 signal transduction by PIAS3. Science. 1997;278(5344):1803-1805.

16. Nallar SC, Kalakonda S, Lindner DJ, et al. Tumor-derived mutations in the gene associated with retinoid interferon-induced mortality (GRIM-19) disrupt its anti-signal transducer and activator of transcription 3 (STAT3) activity and promote oncogenesis. J Biol Chem. 2013;288(11):7930-7941.

17. Zhang X, Guo A, Yu J, et al. Identification of STAT3 as a substrate of receptor protein tyrosine phosphatase T. Proc Natl Acad Sci U S A. 2007;104(10):4060-4064.

18. Veeriah S, Brennan C, Meng S, et al. The tyrosine phosphatase PTPRD is a tumor suppressor that is frequently inactivated and mutated in glioblastoma and other human cancers. Proc Natl Acad Sci U S A. 2009;106(23):9435-9440.

19. Yamamoto T, Sekine Y, Kashima K, et al. The nuclear isoform of protein-tyrosine phosphatase TC-PTP regulates interleukin-6-mediated signaling pathway through STAT3 dephosphorylation. Biochem Biophys Res Commun. 2002;297(4):811-817.

20. Zhang W, Chan RJ, Chen H, et al. Negative regulation of Stat3 by activating PTPN11 mutants contributes to the pathogenesis of Noonan syndrome and juvenile myelomonocytic leukemia. J Biol Chem. 2009;284(33):22353-22363.

21. Nie Y, Erion DM, Yuan Z, et al. STAT3 inhibition of gluconeogenesis is downregulated by SirT1. Nat Cell Biol. 2009;11(4):492-500.

22. Bernier M, Paul RK, Martin-Montalvo A, et al. Negative regulation of STAT3 protein-mediated cellular respiration by SIRT1 protein. J Biol Chem. 2011;286(22):19270-19279.

23. Gao H, Guo R-F, Speyer C, et al. Stat3 activation in acute lung injury. J Immunol. 2004;172(12):7703-7712.

24. Braun DA, Fribourg M, Sealfon SC. Cytokine response is determined by duration of receptor and signal transducers and activators of transcription 3 (STAT3) activation. J Biol Chem. 2013;288(5): 2986-2993.

25. Dauer DJ, Ferraro B, Song L, et al. Stat3 regulates genes common to both wound healing and cancer. Oncogene. 2005;24(21):3397-3408.

26. Gallucci RM, Simeonova PP, Matheson JM, et al. Impaired cutaneous wound healing in interleukin-6-deficient and immunosuppressed mice. FASEB J. 2000;14(15):2525-2531.

27. Sano S, Itami S, Takeda K, et al. Keratinocyte-specific ablation of Stat3 exhibits impaired skin remodeling, but does not affect skin morphogenesis. EMBO J. 1999;18(17):4657-4668.

28. Pickert G, Neufert C, Leppkes M, et al. STAT3 links IL-22 signaling in intestinal epithelial cells to mucosal wound healing. $J$ Exp Med. 2009;206(7):1465-1472. 
29. Coffer PJ, van Puijenbroek A, Burgering BM, et al. Insulin activates Stat3 independently of p21ras-ERK and PI-3 K signal transduction. Oncogene. 1997;15(21):2529-2539.

30. Carvalheira JB, Siloto RM, Ignacchitti I, et al. Insulin modulates leptin-induced STAT3 activation in rat hypothalamus. FEBS Lett. 2001;500(3):119-124.

31. Inoue H, Ogawa W, Asakawa A, et al. Role of hepatic STAT3 in brain-insulin action on hepatic glucose production. Cell Metab. 2006;3(4):267-275.

32. Zong CS, Chan J, Levy DE, Horvath C, Sadowski HB, Wang LH. Mechanism of STAT3 activation by insulin-like growth factor I receptor. J Biol Chem. 2000;275(20):15099-15105.

33. Niso-Santano M, Shen S, Adjemian S, et al. Direct interaction between STAT3 and EIF2AK2 controls fatty acid-induced autophagy. Autophagy. 2013;9(3):415-417.

34. Takeda K, Noguchi K, Shi W, et al. Targeted disruption of the mouse Stat3 gene leads to early embryonic lethality. Proc Natl Acad Sci U SA. 1997;94(8):3801-3804.

35. Sano S, Takahama Y, Sugawara T, et al. Stat3 in thymic epithelial cells is essential for postnatal maintenance of thymic architecture and thymocyte survival. Immunity. 2001;15(2):261-273.

36. Chapman RS, Lourenco PC, Tonner E, et al. Suppression of epithelial apoptosis and delayed mammary gland involution in mice with a conditional knockout of Stat3. Genes Dev. 1999;13(19): 2604-2616.

37. Yu H, Jove R. The STATs of cancer - new molecular targets come of age. Nat Rev Cancer. 2004;4(2):97-105.

38. Cerami E, Gao J, Dogrusoz U, et al. The cBio cancer genomics portal: an open platform for exploring multidimensional cancer genomics data. Cancer Discov. 2012;2(5):401-404

39. Wang Z, Zhu S, Shen M, et al. STAT3 is involved in esophageal carcinogenesis through regulation of Oct-1. Carcinogenesis. 2013; 34(3):678-688.

40. Sen M, Joyce S, Panahandeh M, et al. Targeting Stat3 abrogates EGFR inhibitor resistance in cancer. Clin Cancer Res. 2012;18(18): 4986-4996.

41. Cao X, Tay A, Guy GR, Tan YH. Activation and association of Stat3 with Src in v-Src-transformed cell lines. Mol Cell Biol. 1996;16(4): $1595-1603$

42. Bromberg JF, Horvath CM, Besser D, Lathem WW, Darnell JE. Stat3 activation is required for cellular transformation by v-src. Mol Cell Biol. 1998;18(5):2553-2558.

43. Turkson J, Bowman T, Garcia R, Caldenhoven E, De Groot RP, Jove R. Stat 3 activation by $\mathrm{Src}$ induces specific gene regulation and is required for cell transformation. Mol Cell Biol. 1998;18(5): 2545-2552.

44. Pedranzini L, Leitch A, Bromberg J. Stat3 is required for the development of skin cancer. J Clin Invest. 2004;114(5):619-622.

45. Bowman T, Broome MA, Sinibaldi D, et al. Stat3-mediated Myc expression is required for Src transformation and PDGF-induced mitogenesis. Proc Natl Acad Sci U S A. 2001;98(13):7319-7324.

46. Yu CY, Wang L, Khaletskiy A, et al. STAT3 activation is required for interleukin-6 induced transformation in tumor-promotion sensitive mouse skin epithelial cells. Oncogene. 2002;21(25):3949-3960.

47. Chiarle R, Simmons WJ, Cai H, et al. Stat3 is required for ALKmediated lymphomagenesis and provides a possible therapeutic target. Nat Med. 2005;11(6):623-629.

48. Vultur A, Arulanandam R, Turkson J, Niu G, Jove R, Raptis L. Stat3 is required for full neoplastic transformation by the Simian Virus 40 large tumor antigen. Mol Biol Cell. 2005;16(8):3832-3846.

49. Dasgupta A, Raychaudhuri B, Haqqi T, et al. Stat3 activation is required for the growth of U87 cell-derived tumours in mice. Eur J Cancer. 2009;45(4):677-684.

50. George RE, Sanda T, Hanna M, et al. Activating mutations in ALK provide a therapeutic target in neuroblastoma. Nature. 2008;455(7215): 975-978.
51. Anand M, Lai R, Gelebart P. $\beta$-catenin is constitutively active and increases STAT3 expression/activation in anaplastic lymphoma kinase-positive anaplastic large cell lymphoma. Haematologica. 2011;96(2):253-261.

52. Jones AV, Kreil S, Zoi K, et al. Widespread occurrence of the JAK2 V617F mutation in chronic myeloproliferative disorders. Blood. 2005;106(6):2162-2168.

53. Scott LM, Campbell PJ, Baxter EJ, et al. The V617F JAK2 mutation is uncommon in cancers and in myeloid malignancies other than the classic myeloproliferative disorders. Blood. 2005;106(8):2920-2921.

54. Campbell PJ, Griesshammer M, Döhner K, et al. V617F mutation in JAK2 is associated with poorer survival in idiopathic myelofibrosis. Blood. 2006;107(5):2098-2100

55. Oku S, Takenaka K, Kuriyama T, et al. JAK2 V617F uses distinct signalling pathways to induce cell proliferation and neutrophil activation. Br J Haematol. 2010;150(3):334-344.

56. Fleming TP, Saxena A, Clark WC, et al. Amplification and/or overexpression of platelet-derived growth factor receptors and epidermal growth factor receptor in human glial tumors. Cancer Res. 1992;52(16):4550-4553.

57. Yeh HH, Lai WW, Chen HH, Liu HS, Su WC. Autocrine IL-6-induced Stat3 activation contributes to the pathogenesis of lung adenocarcinoma and malignant pleural effusion. Oncogene. 2006;25(31): 4300-4309.

58. Grandis JR, Drenning SD, Chakraborty A, et al. Requirement of Stat3 but not Stat 1 activation for epidermal growth factor receptor- mediated cell growth In vitro. J Clin Invest. 1998;102(7):1385-1392.

59. Batra SK, Castelino-Prabhu S, Wikstrand CJ, et al. Epidermal growth factor ligand-independent, unregulated, cell-transforming potential of a naturally occurring human mutant EGFRvIII gene. Cell Growth Differ. 1995;6(10):1251-1259.

60. Lo HW, Cao X, Zhu H, Ali-Osman F. Cyclooxygenase-2 is a novel transcriptional target of the nuclear EGFR-STAT3 and EGFRvIIISTAT3 signaling axes. Mol Cancer Res. 2010;8(2):232-245.

61. He B, You L, Uematsu K, et al. SOCS-3 is frequently silenced by hypermethylation and suppresses cell growth in human lung cancer. Proc Natl Acad Sci U S A. 2003;100(24):14133-14138.

62. Iwamoto T, Senga T, Naito Y, et al. The JAK-inhibitor, JAB/ SOCS-1 selectively inhibits cytokine-induced, but not v-Src induced JAK-STAT activation. Oncogene. 2000;19(41):4795-4801.

63. Capello D, Gloghini A, Baldanzi G, et al. Alterations of negative regulators of cytokine signalling in immunodeficiency-related non-Hodgkin lymphoma. Hematol Oncol. 2013;31(1):22-28.

64. Laczmanska I, Karpinski P, Bebenek M, et al. Protein tyrosine phosphatase receptor-like genes are frequently hypermethylated in sporadic colorectal cancer. J Hum Genet. 2013;58(1):11-15.

65. Kou X, Chen N, Feng Z, Luo L, Yin Z. GSTP1 negatively regulates Stat3 activation in epidermal growth factor signaling. Oncol Lett. 2013;5(3):1053-1057.

66. Zhong S, Tang MW, Yeo W, Liu C, Lo YM, Johnson PJ. Silencing of GSTP1 gene by CpG island DNA hypermethylation in HBVassociated hepatocellular carcinomas. Clin Cancer Res. 2002;8(4): $1087-1092$

67. Lin X, Asgari K, Putzi MJ, et al. Reversal of GSTP1 CpG island hypermethylation and reactivation of pi-class glutathione $\mathrm{S}$-transferase (GSTP1) expression in human prostate cancer cells by treatment with procainamide. Cancer Res. 2001;61(24):8611-8616.

68. Schlessinger K, Levy DE. Malignant transformation but not normal cell growth depends on signal transducer and activator of transcription 3. Cancer Res. 2005;65(13):5828-5834.

69. Corvinus FM, Orth C, Moriggl R, et al. Persistent STAT3 activation in colon cancer is associated with enhanced cell proliferation and tumor growth. Neoplasia. 2005;7(6):545-555.

70. Niu G, Bowman T, Huang M, et al. Roles of activated Src and Stat3 signaling in melanoma tumor cell growth. Oncogene. 2002;21(46): 7001-7010. 
71. Rahaman SO, Harbor PC, Chernova O, Barnett GH, Vogelbaum MA, Haque SJ. Inhibition of constitutively active Stat3 suppresses proliferation and induces apoptosis in glioblastoma multiforme cells. Oncogene. 2002;21(55):8404-8413.

72. Bhardwaj A, Sethi G, Vadhan-Raj S, et al. Resveratrol inhibits proliferation, induces apoptosis, and overcomes chemoresistance through down-regulation of STAT3 and nuclear factor-kappaB-regulated antiapoptotic and cell survival gene products in human multiple myeloma cells. Blood. 2007;109(6):2293-2302.

73. Weerasinghe P, Garcia GE, Zhu Q, et al. Inhibition of Stat3 activation and tumor growth suppression of non-small cell lung cancer by G-quartet oligonucleotides. Int J Oncol. 2007;31(1):129-136.

74. Leslie K, Lang C, Devgan G, et al. Cyclin D1 is transcriptionally regulated by and required for transformation by activated signal transducer and activator of transcription 3. Cancer Res. 2006;66(5): 2544-2552.

75. Ewen ME, Lamb J. The activities of cyclin D1 that drive tumorigenesis. Trends Mol Med. 2004;10(4):158-162.

76. Cao X, Liu D, Yan X, et al. Stat3 inhibits WTX expression through up-regulation of microRNA-370 in Wilms tumor. FEBS Lett. 2013;587(6):639-644.

77. Zhuang L, Lee CS, Scolyer RA, et al. Mcl-1, Bcl-XL and Stat3 expression are associated with progression of melanoma whereas Bcl-2, AP-2 and MITF levels decrease during progression of melanoma. Mod Pathol. 2007;20(4):416-426.

78. Catlett-Falcone R, Landowski TH, Oshiro MM, et al. Constitutive activation of Stat3 signaling confers resistance to apoptosis in human U266 myeloma cells. Immunity. 1999;10(1):105-115.

79. Zushi S, Shinomura Y, Kiyohara T, et al. STAT3 mediates the survival signal in oncogenic ras-transfected intestinal epithelial cells. Int $J$ Cancer. 1998;78(3):326-330.

80. Real PJ, Sierra A, De Juan A, Segovia JC, Lopez-Vega JM, Fernandez-Luna JL. Resistance to chemotherapy via Stat3-dependent overexpression of Bcl-2 in metastatic breast cancer cells. Oncogene. 2002;21(50):7611-7618.

81. Timofeeva OA, Tarasova NI, Zhang X, et al. STAT3 suppresses transcription of proapoptotic genes in cancer cells with the involvement of its N-terminal domain. Proc Natl Acad Sci U S A. 2013;110(4):1267-1272.

82. Xie TX, Huang FJ, Aldape KD, et al. Activation of stat3 in human melanoma promotes brain metastasis. Cancer Res. 2006;66(6): 3188-3196.

83. Itoh M, Murata T, Suzuki T, et al. Requirement of STAT3 activation for maximal collagenase-1 (MMP-1) induction by epidermal growth factor and malignant characteristics in T24 bladder cancer cells. Oncogene. 2006;25(8):1195-1204.

84. Fujii M, Honma M, Takahashi H, Ishida-Yamamoto A, Iizuka H. Intercellular contact augments epidermal growth factor receptor (EGFR) and signal transducer and activator of transcription 3 (STAT3)activation which increases podoplanin-expression in order to promote squamous cell carcinoma motility. Cell Signal. 2013;25(4):760-765.

85. Zhou W, Grandis JR, Wells A. STAT3 is required but not sufficient for EGF receptor-mediated migration and invasion of human prostate carcinoma cell lines. Br J Cancer. 2006;95(2):164-171.

86. Priester M, Copanaki E, Vafaizadeh V, et al. STAT3 silencing inhibits glioma single cell infiltration and tumor growth. Neuro-oncology. 2013;15(7):840-852.

87. Cascio S, Ferla R, D'Andrea A, et al. Expression of angiogenic regulators, VEGF and leptin, is regulated by the EGF/PI3K/STAT3 pathway in colorectal cancer cells. J Cell Physiol. 2009;221(1):189-194.

88. Wei LH, Kuo ML, Chen CA, et al. Interleukin-6 promotes cervical tumor growth by VEGF-dependent angiogenesis via a STAT3 pathway. Oncogene. 2003;22(10):1517-1527.

89. Valdembri D, Serini G, Vacca A, Ribatti D, Bussolino F. In vivo activation of JAK2/STAT-3 pathway during angiogenesis induced by GM-CSF. FASEB J. 2002;16(2):225-227.
90. Zhao M, Gao FH, Wang JY, et al. JAK2/STAT3 signaling pathway activation mediates tumor angiogenesis by upregulation of VEGF and bFGF in non-small-cell lung cancer. Lung Cancer. 2011;73(3):366-374.

91. Sriuranpong V, Park JI, Amornphimoltham P, Patel V, Nelkin BD, Gutkind JS. Epidermal growth factor receptor-independent constitutive activation of STAT3 in head and neck squamous cell carcinoma is mediated by the autocrine/paracrine stimulation of the interleukin 6/gp130 cytokine system. Cancer Res. 2003;63(11):2948-2956.

92. Niu G, Wright KL, Huang M, et al. Constitutive Stat3 activity upregulates VEGF expression and tumor angiogenesis. Oncogene. 2002;21(13):2000-2008

93. Masuda M, Ruan HY, Ito A, et al. Signal transducers and activators of transcription 3 up-regulates vascular endothelial growth factor production and tumor angiogenesis in head and neck squamous cell carcinoma. Oral Oncol. 2007;43(8):785-790.

94. Grivennikov S, Karin E, Terzic J, et al. IL-6 and Stat3 are required for survival of intestinal epithelial cells and development of colitisassociated cancer. Cancer Cell. 2009;15(2):103-113.

95. Liang J, Nagahashi M, Kim EY, et al. Sphingosine-1-phosphate links persistent STAT3 activation, chronic intestinal inflammation, and development of colitis-associated cancer. Cancer Cell. 2013;23(1):107-120

96. Burdelya L, Kujawski M, Niu G, et al. Stat 3 activity in melanoma cells affects migration of immune effector cells and nitric oxide-mediated antitumor effects. J Immunol. 2005;174(7):3925-3931.

97. See AP, Han JE, Phallen J, et al. The role of STAT3 activation in modulating the immune microenvironment of GBM. J Neurooncol. 2012;110(3):359-368.

98. Sherry MM, Reeves A, Wu JK, Cochran BH. STAT3 is required for proliferation and maintenance of multipotency in glioblastoma stem cells. Stem Cells. 2009;27(10):2383-2392.

99. Kim Y, Hsu J, Zhou T, et al. Potent in vivo pharmacology of AZD9150, a next-generation, constrained ethyl-modified antisense oligonucleotide targeting STAT3 in multiple preclinical cancer models [abstract]. In: Proceedings of the 104th Annual Meeting of the American Association for Cancer Research; 2013 Apr 6-10; Washington, DC. Philadelphia (PA): AACR; 2013. Abstract nr LB-317

100. Sen M, Thomas SM, Kim S, et al. First-in-human trial of a STAT3 decoy oligonucleotide in head and neck tumors: implications for cancer therapy. Cancer Discov. 2012;2(8):694-705.

101. Schust J, Sperl B, Hollis A, Mayer TU, Berg T. Stattic: a smallmolecule inhibitor of STAT3 activation and dimerization. Chem Biol. 2006;13(11):1235-1242.

102. Ren Z, Cabell LA, Schaefer TS, McMurray JS. Identification of a high-affinity phosphopeptide inhibitor of Stat3. Bioorg Med Chem Lett. 2003;13(4):633-636.

103. Zhang X, Yue P, Fletcher S, Zhao W, Gunning PT, Turkson J. A novel small-molecule disrupts Stat3 SH2 domain-phosphotyrosine interactions and Stat3-dependent tumor processes. Biochem Pharmacol. 2010;79(10):1398-1409.

104. Gomez C, Bai L, Zhang J, et al. Design, synthesis, and evaluation of peptidomimetics containing Freidinger lactams as STAT3 inhibitors. Bioorg Med Chem Lett. 2009;19(6):1733-1736.

105. Epling-Burnette PK, Liu JH, Catlett-Falcone R, et al. Inhibition of STAT3 signaling leads to apoptosis of leukemic large granular lymphocytes and decreased Mcl-1 expression. J Clin Invest. 2001;107(3):351-362.

106. Song H, Wang R, Wang S, Lin J. A low-molecular-weight compound discovered through virtual database screening inhibits Stat 3 function in breast cancer cells. Proc Natl Acad Sci U S A. 2005;102(13): 4700-4705.

107. Sen M, Tosca PJ, Zwayer C, et al. Lack of toxicity of a STAT3 decoy oligonucleotide. Cancer Chemother Pharmacol. 2009;63(6):983-995.

108. Looyenga BD, Hutchings D, Cherni I, Kingsley C, Weiss GJ, Mackeigan J. STAT3 is activated by JAK2 independent of key oncogenic driver mutations in non-small cell lung carcinoma. PLoS ONE. 2012;7(2):e30820. 
109. Korkaya H, Kim GI, Davis A, et al. Activation of an IL6 inflammatory loop mediates trastuzumab resistance in HER2+ breast cancer by expanding the cancer stem cell population. Mol Cell. 2012;47(4):570-584.

110. Bewry NN, Nair RR, Emmons MF, Boulware D, Pinilla-Ibarz J, Hazlehurst LA. Stat3 contributes to resistance toward BCR-ABL inhibitors in a bone marrow microenvironment model of drug resistance. Mol Cancer Ther. 2008;7(10):3169-3175.
111. Marcher-Bauer A, Lu S, Anderson JB, et al. CDD: a Conserved Domain Database for the functional annotation of proteins. Nuclieic Acids Res. 2011:39; 225-229.

\section{Publish your work in this journal}

OncoTargets and Therapy is an international, peer-reviewed, open access journal focusing on the pathological basis of all cancers, potential targets for therapy and treatment protocols employed to improve the management of cancer patients. The journal also focuses on the impact of management programs and new therapeutic agents and protocols on

\section{Dovepress}

patient perspectives such as quality of life, adherence and satisfaction. The manuscript management system is completely online and includes a very quick and fair peer-review system, which is all easy to use. Visit http://www.dovepress.com/testimonials.php to read real quotes from published authors. 